\title{
Corela
}

Cognition, représentation, langage

6-1 | 2008

Vol. $6, n^{\circ} 1$

\section{Sur la structure argumentale de la forme faire + infinitif}

\section{Malika Kaheraoui}

\section{OpenEdition}

\section{Journals}

Édition électronique

URL : http://journals.openedition.org/corela/247

DOI : $10.4000 /$ corela. 247

ISSN : $1638-573 \mathrm{X}$

\section{Éditeur}

Cercle linguistique du Centre et de l'Ouest - CerLICO

Référence électronique

Malika Kaheraoui, « Sur la structure argumentale de la forme faire + infinitif », Corela [En ligne],

6-1 | 2008, mis en ligne le 24 juin 2008, consulté le 02 mai 2019. URL : http://journals.openedition.org/ corela/247; DOI : 10.4000/corela.247

Ce document a été généré automatiquement le 2 mai 2019.

\section{(c) (i) (2)(2)}

Corela - cognition, représentation, langage est mis à disposition selon les termes de la licence Creative Commons Attribution - Pas d'Utilisation Commerciale - Partage dans les Mêmes Conditions 4.0 International. 


\title{
Sur la structure argumentale de la forme faire + infinitif
}

\author{
Malika Kaheraoui
}

\section{Introduction}

1 Avec la forme faire+infinitif, dite "factitive" ou "causative"1, on se trouve sans doute confronté à l'un des problèmes les plus complexes en linguistique, que ce soit au niveau syntaxique ou sémantique. C'est surtout la complexité syntaxique de la construction factitive qui a fait l'objet d'une pluralité de travaux. Les cadres chomskyens en offrent un exemple (cf. Kayne, 1977, Zubizarreta, 1986), mais on trouve d'autres types d'analyses, notamment dans le cadre de la Grammaire fonctionnelle de Bresnan (1982), ou dans celui de la grammaire syntagmatique guidée par les têtes (HPSG) de Pollard et Sag (1987). Après une présentation sommaire des propriétés syntaxiques générales de la forme faire + infinitif, nous proposons d'analyser la structure argumentale de cette forme. L'examen des restrictions de sélection sur les arguments sujet et objet permet de rendre compte en effet d'un fonctionnement "bivalent" de cette construction aux niveaux syntaxique et sémantique. Nous examinerons ainsi le rôle de Causateur joué par l'argument-sujet et nous mettrons en relief la particularité de la forme factitive en analysant les rapports entretenus respectivement par le sujet de faire et le sujet de l'infinitif.

\section{Propriétés syntaxiques}

2 La complexité syntaxique de la construction factitive résulte de la fusion de deux prédicats : faire suivi d'un infinitif direct. Les caractéristiques de cette " prédication composite ", comme la décrit Roegiest (1982-83), confèrent à cette construction en français un statut différent par rapport aux autres constructions infinitives. En français, l'union ou la montée de l'infinitif est obligatoire derrière faire et facultative derrière 
laisser et les verbes de perception comme regarder, entendre, etc., considérés aussi comme des factitifs :

(1) Je fais pleurer Marie(1a)*Je fais Marie pleurer

(1b) Je la fais pleurer

(2) J'ai fait renvoyer cette fille

(2a)*'ai fait cette fille renvoyer

(2b) Je l'ai fait renvoyer

(3) Je laisse/entends pleurer Marie

(3a) Je laisse/entends Marie pleurer

3 Le verbe faire ne tolère donc pas d'être séparé du verbe infinitif. Cet infinitif est suivi luimême d'un NP $(1,2)$ qui peut être pronominalisé, comme en $(1 b-2 b)$ par un clitique accusatif, si bien qu'il semble qu'on a affaire à un complément d'objet. Mais ce qui est particulier, c'est que les clitiques s'attachent au verbe faire conjugué et non à l'infinitif, comme c'est le cas avec les constructions infinitives où le verbe n'est pas causatif comme en (4) et (5) et dans lesquelles le pronom clitique s'antépose nécessairement à l'infinitif :
(4) Je veux lire ce livre
(4a) Je veux le lire / *Je le veux lire
(5) Je vais lire ce livre
(5a) Je vais le lire $/ *$ Je le vais lire

4 Dans (1) et (2), le NP semble donc fonctionner comme complément d'objet de la phrase entière et non pas seulement de l'infinitif. Toutefois, un examen de ces énoncés montre que le parallélisme n'est qu'apparent. Les NP postverbaux Marie et cette fille n'ont pas le même statut, comme le montre leur placement respectif dans d'autres structures infinitives :

(1c) *Je vais pleurer Marie

(2c) Je vais renvoyer cette fille

5 Cette fille a réellement le statut de complément d'objet par rapport au verbe infinitif renvoyer alors que Marie est en fait le sujet du verbe intransitif pleurer. Donc, (1) et (2) représentent deux structures différentes : dans la première, le NP postverbal est le sujet sémantique de l'infinitif alors que dans la seconde, ce NP est l'objet sémantique de l'infinitif tandis que le sujet n'est pas exprimé.

6 Le fait que l'infinitif après faire puisse garder son sujet isole la forme factitive parmi les autres constructions infinitives. En effet, ces constructions peuvent être analysées en français comme des phrases S' dont le sujet n'a pas de réalisation lexicale et dont la position de Complémenteur n'est pas nécessairement occupée. Ce sujet nul reçoit néanmoins une interprétation : il est identique à l'un des arguments du verbe introducteur et il peut avoir pour antécédent soit le sujet (6), soit l'objet (7), soit un complément prépositionnel (8) :

(6) Paul veut partir

(7) Je persuade Paul de partir

(8) Je permets à Paul de partir

7 Mais si dans la construction factitive, l'infinitif peut garder son sujet, celui-ci se trouve en position postverbale ; cette position étant en contradiction avec la position canonique d'un sujet par rapport à son verbe. On peut reconnaître là un cas d'inversion du sujet, mais cette disjonction entre la fonction de sujet et la position canonique du sujet est soumise à des conditions, en particulier au principe de "préservation des structures ", qui exclut toute séquence du type V NP NP. Selon ce principe, un constituant ne peut apparaître que dans une position étiquetée correspondant à sa catégorie. Si la place 
postverbale étiquetée NP n'est pas occupée par un complément d'objet par exemple, alors un NP peut s'y trouver sans pour autant remplir la fonction liée à cette position.

Ainsi, si la construction infinitive résulte de l'enchâssement d'une phrase dans une autre phrase, la façon dont cet enchâssement se réalise dépend de la valence du verbe enchâssé. Si le verbe infinitif enchâssé est intransitif, comme travailler en (9), le NP sujet suit l'infinitif et prend la place d'un complément d'objet dans la phrase factitive (9a); si le verbe enchâssé est transitif comme balayer dans (10), le NP objet de la phrase de départ reste à sa place canonique à droite du verbe, alors que le NP sujet prend la forme soit d'un complément en à (10a), qui se comporte d'ailleurs comme un complément datif, ce dont rend compte sa pronominalisation en (10b), soit d'un complément en par (10c). L'infinitif suit toujours immédiatement faire, aucun des deux NP ne peut s'intercaler en effet entre faire et l'infinitif.
(9) Pierre travaille
(9a) Jean fait travailler Pierre
(10) Jean balaie la cour
(10a) Marie fait balayer la cour à Pierre
(10b) Marie lui fait balayer la cour
(10c) Marie fait balayer la cour par Pierre

En réalité, la construction faire+infinitif peut recevoir deux "lectures syntaxiques" différentes. Elle peut être effectivement le résultat de l'enchâssement d'une phrase sous faire et, dans ce cas, faire sera considéré comme un verbe autonome qui prend pour argument-objet une proposition enchâssée infinitive. Mais faire peut être également considéré comme un auxiliaire d'un type spécial qui se combine avec le verbe infinitif pour former un verbe complexe qui prend alors pour argument-objet, l'argument-sujet de l'infinitif, si celui-ci est un verbe intransitif, ou son argument-objet s'il est transitif. Nous reviendrons sur les arguments qui sous-tendent ces deux lectures plus loin (cf. 3.2).

On peut donc voir que l'analyse détaillée de la structure argumentale de faire+infinitif permettrait de rendre compte du fonctionnement syntaxique de cette forme en lien avec une analyse sémantique effectuée en termes de cadres de rôles thématiques.

\section{Les restrictions de sélection sur les arguments sujet et objet de faire + infinitif}

\subsection{Un argument-sujet non restreint}

11 L'enchâssement de faire dans une autre phrase permet d'introduire un nouvel argument qui s'ajoute aux arguments déjà présents du verbe enchâssé et qui portera selon nous le rôle de Causateur (cf. 4.1). On peut remarquer que cet argument-sujet est non restreint. Il peut référer en effet à des humains (12), à des objets concrets (14-15), à des forces naturelles (13), à des événements (16), à des états psychologiques (11-17-18) :

(11) Il s'en souvenait quand cette vieille douleur lui faisait porter une main à la nuque.

(12) Le colonel Lejeune déboula sur la place et fit cabrer son cheval devant Masséna.

(13) Première nuit, un vent contraire, qui amenait une odeur de brûlé, agaçait les chevaux et faisait voleter les plumes sur le bicorne du maréchal.

(14) Son livre m'a fait rêver, et je m'y suis jetée avec cet espoir bizarre que nourrissent, je suppose, les autres aussi bien que moi : trouver dans un livre LA réponse à une question dont on n'a pas la moindre idée. 
(15) La cavalerie défilait entre les torchères qui faisaient luire les armes et les parements d'uniformes.

(16) Il allait atteindre la moitié du pont lorsque des hurlements le firent tressaillir.

(17) Pendant ce temps, la stupidité de cette bataille le faisait frémir de colère.

(18) Tant de cruauté m'a fait sourire.

(19)(...) Cela m'a fait pleurer dans mon demi- sommeil.

12 Nous partons de l'hypothèse que la variété des types de sujets peut cacher une description unifiée et que cet argument a une interprétation prédicative. En effet, cette interprétation peut être obtenue pour les sujets qui renvoient à des événements ou des états et qui correspondent au mieux à des nominalisations (hurlements (16), stupidité (17), cruauté (18)) ou à des arguments propositionnels, quand la nominalisation n'est pas possible. (11) peut être paraphrasé par (11a). (19) présente un anaphorique précédant une proposition (pas notée), un contexte proche de celui des arguments propositionnels (19b) :

(11a) Il s'en souvenait quand le fait de ressentir cette vieille douleur lui faisait porter une main à la nuque

(19a) L'idée qu'elle ait agi ainsi m'a fait pleurer dans mon demi-sommeil.

Concernant vent dans l'exemple (13), il fait partie des événements de type « météorologique » ou des "forces naturelles ». Ces noms se comportent comme des nominalisations. Selon Vendler (1967 : 141), ils peuvent en effet entrer dans le contexte d'un conteneur comme to cause, sans suggérer la restitution d'une nominalisation ${ }^{2}$.

Quand le sujet réfère à un objet concret, comme en (14) et (15), on peut l'analyser comme un prédicat elliptique qui dénote une propriété sous forme d'une nominalisation du type (15a) et (14a) ou d'une dérivation métonymique ${ }^{3}$ qui peut mettre en relation, entre autres, le contenant et le contenu (14b) :

(14a) La lecture de son livre m'a fait rêver.

(14b) Le contenu de son livre m'a fait rêver.

(15a) La luminosité des torchères faisait luire les armes et les parements

d'uniformes

Que cette variété de sujets puisse recevoir une interprétation propositionnelle corrobore l'idée qu'une entité de premier ordre ne peut constituer une cause. Un objet concret ou un humain ne sont des causateurs que de manière indirecte : un objet l'est par l'intermédiaire d'une propriété qui lui est associée, un humain l'est soit par l'intermédiaire d'une propriété ou d'une action qu'il effectue. Autrement dit, avec un sujet humain, la causativité sera confrontée à la notion d'agentivité (cf.4.1). L'exemple (12) peut dans ce sens être paraphrasé par (12a) et le sujet humain s'analysera alors également comme une prédication elliptique :

(12a) Le colonel Lejeune a fait quelque chose/a quelque chose en lui qui a la vertu de faire cabrer son cheval. Toutefois, cette paraphrase ne fonctionne pas dans le cas où le verbe infinitif est transitif et que l'enchâssement de faire s'accompagne d'un déplacement du NP sujet du verbe infinitif enchâssé en position de complément en par ou en à, comme le suggèrent ces exemples que nous reprenons :

(20) Marie a fait balayer la cour par Pierre

(20a)* Marie a fait quelque chose/a quelque chose en elle qui a la vertu de faire balayer la cour par Pierre

(21) Jean a fait lire un roman à Pierre

(21a)* Jean a fait quelque chose/a quelque chose en lui qui a la vertu de faire lire un roman à Pierre 
Cela laisse supposer, à notre avis, que l'examen de la nature de l'argument Causateur humain dans la construction factitive doit être effectuée en prenant en compte la transitivité du verbe infinitif ainsi que la relation que peut entretenir l'individu dénoté par le sujet de faire (le Causateur) et celui dénoté par le sujet du verbe infinitif, quand il est introduit par les prépositions à ou par. Nous reviendrons donc sur les restrictions de sélection de l'argument-sujet de faire après l'examen du rôle thématique Causateur que nous lui attribuons (cf. 4).

\subsection{L'argument-objet : deux lectures de faire+infinitif}

$\mathrm{Au}$ niveau de l'argument-objet, se pose le problème expliqué plus haut sur la « lecture syntaxique » à donner à la structure faire + infinitif. Les deux lectures isolent soit un verbe autonome faire qui prend pour argument-objet une proposition enchâssée infinitive (22a), soit un complexe verbal faire+infinitif qui prend pour argument-objet, l'argument-sujet de l'infinitif (22b), si celui-ci est un verbe intransitif, ou son argumentobjet s'il est transitif (23):

(22) Paul a fait travailler Jean

(22a) [Paul faire [Jean travailler]]

(22b) [Paul [faire travailler] Jean]

(23) [Je [faire soigner] le malade]

Les arguments fournis pour telle ou telle position dépendent souvent des cadres théoriques adoptés. Dans le cadre transformationnel du type Aspects, Kayne (1977) propose de dériver des phrases à structure factitive comme $(20,21,22)$ des structures profondes $(20 \mathrm{~b}, 21 \mathrm{~b}, 22 \mathrm{c})$, où les NP sujets seraient effectivement en position sujet du verbe qui les précède en structure de surface ${ }^{4}$. Comme les règles d'interprétation sémantique qui assignent des fonctions thématiques aux divers arguments d'un prédicat seront définis dans la structure profonde, elles s'appliqueront avant que les NP ne soient déplacés hors de la position sujet. De ce fait, quelle que soit la structure dérivée de ces phrases, leur structure profonde sera formée de deux propositions où faire sera le verbe de la proposition principale et le verbe à l'infinitif celui de la subordonnée dans laquelle sujet et objet gardent leur position canonique :
(20b) [S Marie a fait[S Pierre balayer la cour]]
(21b) [S Jean a fait[S Pierre lire un roman]]
(22c) [S Paul a fait[S Jean travailler]]

Autrement dit, Kayne postule que les phrases à structure factitive sont dérivées d'une structure sous-jacente dans laquelle faire est suivi d'un complément phrastique, plus une transformation qui change l'ordre relatif du sujet enchâssé et du verbe. Cette transformation est appelée faire + infinitif (FI) ; elle est formulée comme suit :

$\mathrm{FI}: \mathrm{X}$ - faire - NP - V - (NP) - Y > 124536

$$
\begin{array}{llllll}
1 & 2 & 3 & 4 & 5 & 6
\end{array}
$$

Appliquée à (21b) par exemple, on aura :

FI : Jean fait - NP[Pierre] - [lire] - [un roman]

$\rightarrow>$ Jean fait [lire] [un roman] NP[Pierre]

Le NP situé entre faire et l'infinitif est placé à droite de l'objet non-prépositionnel de l'infinitif. Pour remédier à l'agrammaticalité de la phrase produite (21c), une seconde transformation sera appliquée appelée insertion de à (INS-A) et qui consiste à insérer 
obligatoirement la préposition à devant le sujet déplacé de l'infinitif, quand ce dernier est suivi d'un NP (cf. Kayne, 1977 : 199) :

(21c)*Jean a fait lire un roman Pierre

INS-A : X - faire - V - NP - NP - Y -> 1234 à 56

$$
\begin{array}{llllll}
1 & 2 & 3 & 4 & 5 & 6
\end{array}
$$

Cette transformation a une contrepartie qui s'en approche et qui fait apparaître la préposition par. Selon Kayne, la présence de par suggère que la construction faire par peut être mise en rapport avec le passif. En se basant sur l'analyse proposée par Chomsky (1965), il propose de représenter la dérivation de phrases au passif sous la forme par $\Delta$ de sorte que la dérivation d'une phrase au passif comme (24) soit (24a) :

(24) Ce roman est lu par Pierre

(24a) Pierre lire - ce roman - par $\Delta$

-> ce livre - être + é - lire - par Paul

Ce qui donne pour la forme factitive en faire par la dérivation faire par suivante obtenue par application de la transformation dite "FP"5:

(24b) Jean fait Pierre - lire - un roman - par $\Delta$

$\rightarrow$ Jean fait lire - un roman - par Pierre

FP : X faire NP V Y par $\rightarrow$ Z -> 1245638

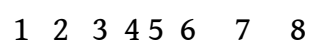

Adoptant le cadre de la théorie lexicaliste stricte de Bresnan (1976-1977), Morin (1978) s'oppose à l'approche transformationnelle proposée par Kayne. Il considère en effet que les constructions factitives sont engendrées telles quelles dans la base et non pas dérivées d'une structure sous-jacente à " deux étages ". Autrement dit, ces constructions ne contiennent pas, selon lui, de proposition enchâssée et faire est considéré comme étant un auxiliaire d'un type spécial qui forme avec le verbe enchâssé un verbe complexe.

Sans défendre Kayne, Reed (1991) se base sur la structure thématique assignée aux causatives en français pour rejeter la proposition de Morin. En effet, un énoncé comme Paul a fait travailler Jean est problématique pour les syntacticiens qui travaillent dans la le modèle de GB (Government-Binding) et plus précisément au niveau de la théorie thématique (Theta Theory), parce que le sujet du verbe enchâssé (Jean), peut être interprété à la fois comme argument thématique du verbe travailler, mais aussi du causatif faire, dans le sens où ce sujet accomplit l'action dénotée par le verbe enchâssé travailler tout en étant amené par le sujet de faire (Paul) à le faire. Ce « double marquage thématique » est strictement interdit par le principe de la Theta Theory ( $\theta$-Criterion), ainsi que par celui de Extended Projection Principle (EPP) ${ }^{6}$. Ces deux principes stipulent en effet qu'un argument doit être associé à un et un seul rôle thématique et ce rôle thématique reste constant à tous les niveaux de représentation.

Pour Kayne, étant donné que la causative se présente comme une structure à deux étages avec une proposition principale en faire et une proposition subordonnée enchâssée infinitive, le sujet de la subordonnée enchâssée reçoit un seul rôle thématique, celui d'argument externe ${ }^{7}$ attaché au prédicat enchâssé. Autrement dit, Jean est interprété uniquement comme l'Agent qui accomplit l'action de travailler. Cependant, on ne peut pas nier que le sujet de faire entre dans une certaine relation avec le sujet enchâssé, (Paul fait en sorte que Jean travaille), ce qui autoriserait à dire que, d'un point de vue thématique, faire et le verbe enchâssé marqueraient doublement le sujet enchâssé, à la fois comme argument externe et comme argument interne. Selon Reed (1991), ce double 
marquage n'est pas possible et le fait que l'on puisse interpréter le sujet enchâssé comme un argument interne du causatif faire est dû à un aspect pragmatique relatif à nos connaissances du monde réel. C'est ainsi qu'elle voit la différence entre une classe de causatifs en anglais interprétés comme étant sémantiquement complexes comme walk, feed, etc., et la forme factitive faire + infinitif dans les exemples suivants ${ }^{8}$ :

(25) John walked the dog

(25a) Jean a fait marcher le chien

(26) John walked the dog, well, actually the dog didn't walk since John carried him in his arms

(26a)* Jean a fait marcher son chien, mais en fait son chien n'a pas marché parce qu'il l'a porté dans ses bras

La même analyse du double marquage thématique peut être proposée pour the dog en (25) : il peut recevoir à la fois le rôle thématique d'Agent (le chien marche) et un rôle thématique interne (Jean a fait en sorte que le chien marche). Cependant, alors qu'en anglais, on peut admettre un contexte dans lequel l'objet enchâssé du verbe causatif ne peut être interprété comme Agent, comme le suggère (26), l'agrammaticalité de la traduction française avec faire + infinitif en (26a) suggère le contraire. En se basant sur le principe de la Theta Theory, Reed pense que l'objet du causatif walk en anglais est un argument interne ; à aucun niveau il ne peut être interprété comme un argument externe. Le fait de considérer que the dog est aussi un agent de l'action de marcher est une interprétation de type pragmatique : en général quand on sort le chien pour le faire marcher, on s'attend à ce qu'il marche. Par contre, la construction factitive en français suggère que le sujet enchâssé son chien est l'argument externe du prédicat enchâssé seulement et cela à tous les niveaux de dérivation. Mais cela n'explique pas, nous semble$\mathrm{t}$-il, le lien qui existe entre les deux sujets de faire et de l'infinitif, et cette relation sémantique ne peut pas être réduite à un simple point de vue pragmatique. Quand on fait marcher un chien, il est vrai qu'on s'attend à ce qu'il marche, mais si on le fait marcher, c'est qu'on exerce une sorte de coercition sur lui, puisqu'on fait en sorte qu'il marche. Ces deux interprétations ne sont pas exclusives l'une de l'autre ; au contraire, elles s'impliquent.

37 Une autre analyse est proposée par Zaenen \& Dalrymple (1996) dans le cadre de la Grammaire Lexicale Fonctionnelle ${ }^{9}$. Ces auteurs admettent que la construction factitive en français met en jeu la formation d'un prédicat unique d'un point de vue syntaxique. Mais si de ce point de vue, la forme factitive semble simple (ou simplifiée), il en est autrement au niveau sémantique. Les auteurs posent que cette forme en français est caractérisée par une structure fonctionnelle (structure-f) simple, sans enchâssement, liée à une représentation sémantique complexe. La structure-f est une structure de traits qui code directement les fonctions grammaticales. Cette structure est construite à partir de la structure en constituants (structure-c) qui représente l'organisation superficielle et dans laquelle le verbe causatif faire est présenté comme un verbe principal qui prend un complément VP. Dans ce modèle, c'est la structure-f qui constitue la représentation syntaxique cruciale. La construction factitive présente alors un double problème architectural : celui de représenter la relation entre la structure-f et la sémantique et, celui de représenter la relation entre la structure-c et la structure-f.

Dès lors, l'objectif des auteurs est d'expliquer comment faire se combine avec un verbe de telle ou telle valence en autorisant une structure monophrastique correcte. Dans un exemple comme Pierre fait venir Paul, Zaenen \& Dalrymple (1996 : 88) soutiennent que faire demande deux arguments : d'abord un argument dont le rôle est Causateur (X), 
c'est-à-dire le rôle rempli par Pierre, ensuite une interprétation préliminaire $(\mathrm{P})$ pour la structure-f à laquelle il est associé. Cette interprétation sera présente quand le verbe infinitif venir sera fourni avec ses arguments. Autrement dit, faire demande que le Causateur $(\mathrm{X})$ et l'interprétation préliminaire $(\mathrm{P})$ soient consommées afin de produire une nouvelle interprétation : cause $(\mathrm{X}, \mathrm{P})$. Ainsi, sont assignées à la projection sémantique de la structure-f deux interprétations sémantiques différentes : $P$, l'interprétation associée au verbe infinitif, puis cause $(\mathrm{X}, \mathrm{P})$ dans laquelle $\mathrm{P}$ est un argument de la relation cause portée par faire.

Les auteurs ajoutent une autre projection qui a pour rôle d'associer les fonctions grammaticales de sujet et d'objet aux rôles thématiques de Causateur et d'Agent. Cette projection s'effectue indépendamment du fait que ces rôles soient associés à un seul verbe ou à plus d'un verbe, ce qui est le cas des prédicats complexes. A ce moment-là, le sujet et l'objet qui appartiennent à la même structure-f peuvent être associés à des rôles attribués par des prédicats différents. C'est le cas de faire + infinitif : le rôle de Causateur est attribué par faire, celui d'Agent par l'infinitif.

On peut alors remarquer que dans ce système, on a un verbe causatif faire avec un premier argument Causateur et un deuxième argument propositionnel. Cette représentation sémantique est indifférente au nombre des arguments du verbe infinitif. Elle requiert seulement que ce verbe ait un Agent et répond à une intuition concernant la classe des verbes qui se combinent avec faire. Ce système présente d'un côté une unicité syntaxique du prédicat sous la forme d'un prédicat complexe, et d'un autre côté, une représentation sémantique complexe où le sens du complexe verbal est prédit à partir du sens du verbe causatif faire qui se combine avec le sens de l'infinitif associé. Le résultat de la combinaison est un prédicat qui a autant d'arguments que l'infinitif plus un nouvel argument : le Causateur. Le modèle admet alors une séparation entre syntaxe et sémantique pour l'analyse de faire + infinitif : faire en se combinant avec un verbe, qu'il soit transitif ou intransitif, en modifie seulement la structure argumentale, la valeur du prédicat complexe reste celle de l'infinitif.

\subsection{Le fonctionnement "bivalent" de faire}

41 Ces approches témoignent toutes de la complexité des relations entre syntaxe et sémantique dans l'analyse de la structure faire + infinitif. A cet égard, l'analyse proposée par Zaenen \& Dalrymple reste la plus séduisante. En faisant correspondre une représentation syntaxique simplifiée sans enchâssement à une représentation sémantique où faire se comporte comme un verbe principal, les auteurs arrivent en effet à rendre compte du comportement "bivalent" de la structure factitive. Car, ce verbe a bien, à notre avis, un comportent double qui se manifeste aux niveaux syntaxique et sémantique.

D'un point de vue syntaxique, il est plus adéquat en effet de considérer que faire forme avec le verbe infinitif un complexe verbal. Le comportement des clitiques, accusatif ou datif, est à ce point de vue éloquent : les pronoms clitiques s'antéposent nécessairement à ce complexe verbal (Il l'a fait travailler ; Il lui a fait lire un roman), comme c'est le cas pour un verbe à arguments sujet, objet direct/indirect (Il la mange, Il lui plaît). Nous avons vu qu'à la différence des autres structures infinitives, aucun NP ne peut s'intercaler entre faire et son infinitif. 
43 En revanche, l'analyse sémantique effectuée en termes de cadres de rôles nécessite de mettre en relation les rôles thématiques assignés respectivement au sujet de faire et à celui du verbe infinitif. On ne peut pas nier en effet l'existence d'une relation entre les deux sujets. D'un autre côté, rappelons que c'est au niveau de l'argument-objet que s'exprime le changement produit sous l'effet du Causateur. On sait que l'expression de la causation est en corrélation étroite avec la notion de changement. Celle-ci se manifeste en effet comme un "prérequis" de la causation : à toute cause, il y a un changement produit, affectant un patient. On peut comparer en ce sens faire à d'autres verbes causatifs, mais qui n'ont pas la même structure argumentale, ni le même mode d'expression de la causation et du changement. Nous prenons le cas de détruire et de causer (cf. kaheraoui, 2002).

Les deux rejoignent faire dans la sélection de l'argument-sujet, puisqu'il est également non restreint et accepte l'interprétation propositionnelle. Les différences surgissent quand cet argument-sujet est humain. Détruire (27) a pour sujet un Agent qui est aussi Causateur par l'intermédiaire de l'action accomplie, et pour objet un patient qui est affecté par le changement produit. En revanche, causer (28) fonctionne comme un verbe relateur qui fait le lien entre l'expression de la causation (le sujet humain est uniquement Causateur par l'intermédiaire d'une action accomplie ou d'une propriété qui lui est associée. (f.4.1) et l'expression du changement subi par un patient. En ce sens, ce verbe ne prédique pas la causation de changement entre un argument Agent et un argument Patient, comme pour détruire, mais entre un événement et l'expression du changement subi par un patient. Sur le plan syntaxique, causer se présente selon les termes de Vendler, comme un double "conteneur", puisqu'il appelle deux arguments à interprétation propositionnelle (nominalisations ou argument propositionnel pour le sujet et nominalisation pour l'objet).

(27) Pierre a détruit toutes ses photos

(28) Son fils lui a causé beaucoup de soucis

Pour faire, on peut alors se demander s'il prend pour objet un patient et on considère alors qu'on a affaire à un complexe verbal faire+infinitif qui se comporte comme détruire sur le plan sémantique, soit on a l'expression du changement notée par une proposition infinitive et qui fait apparaître, à la différence de la première analyse, le rôle du sujet de l'infinitif dans l'expression de ce changement. Puisque, comme on va le voir dans le point suivant, ce sujet n'est pas un simple patient qui subit l'action causatrice du sujet de faire. Autrement dit, la caractérisation de la structure argumentale de faire dépend de celle du verbe infinitif enchâssé. Celle-ci représente le deuxième argument du processus de causation représenté par faire. On peut alors établir le parallèle entre causer et faire dans les exemples ci-dessous. Si le verbe causer appelle en position d'argument-objet une nominalisation, faire appelle une proposition infinitive. L'effet du processus causatif déclenché par cet homme est que le soldat a été arrêté. La production de changement dans les deux cas se caractérise par la consommation d'une relation prédicative [ce soldat a été arrêté].

(29) Cet homme a causé l'arrestation de ce soldat

(29a) Cet homme a causé [on a arrêté ce soldat]

(30) Cet homme a fait arrêter ce soldat

(30a) Cet homme a fait [on a arrêté ce soldat]

La particularité de la forme faire+infinitif est qu'on a dans l'expression du changement un agent intermédiaire qui peut être exprimé explicitement ou implicitement selon la 
valence du verbe à l'infinitif. Autrement dit, le sujet Causateur de "faire" a besoin de l'agentivité du sujet de l'infinitif pour produire le changement que cet agent lui-même subit ou fait subir.

\section{L'argument causateur de faire}

\subsection{Causativité, agenivité, factitivité}

47 Pour définir le rôle thématique de Causateur, nous le distinguons de celui d'Agent. Plusieurs conceptions ont tenté de préciser les rapports entre agentivité et causativité. Celle de Dowty (1991) les réunit selon un modèle prototypique qui considère les rôles thématiques comme des concepts qui peuvent être assignés aux arguments à des degrés. L'Agent prototypique de Dowty ${ }^{10}$ est à la fois Agent et Causateur. C'est un humain qui participe volontairement à une action par l'intermédiaire de laquelle il cause un événement ou un changement d'état. La conception de François (1989-1990) soutient au contraire l'autonomie de l'agentivité et de la causativité considérés comme des classificateurs conceptuels des prédications verbales. Cette autonomie impose de distinguer l'Agent, caractérisé par le trait [+agentif] et défini comme un individu animé exerçant une activité non causatrice (courir) ou accomplissant une action causatrice (battre le record du monde) ou préservatrice (conserver son titre), du Causateur spécifié par le trait [+causatif] et représenté par une instance, une force, un événement, une activité ou un agent entraînant le changement subi par un patient. La combinaison entre les deux traits [+causatif], [+agentif] permet de spécifier des actions causatrices qui ont dans leur cadre de rôles un Agent Causateur, c'est-à-dire un animé qui contrôle ses agissements lesquels causent un changement d'état du monde.

Dans l'expression de l'agentivité, la notion de contrôle se confond chez François (1996) avec celle d'intentionnalité. Ainsi, la prise en compte du jugement d'intentionnalité présuppose selon cet auteur la définition de deux types d'Agent : un Agent [+intentionnel] représenté par un animé qui contrôle un procès dont l'aboutissement est conforme à la représentation que cet animé s'en faisait dans une situation initiale et un Agent [intentionnel] qui n'a aucun contrôle sur le procès dont l'aboutissement ne peut être que différent de la représentation que cet Agent pourrait en avoir faite dans une situation initiale. La relation entre intentionnalité et causativité est plus ambiguë. La combinaison des propriétés d'agentivité et d'intentionnalité au trait conceptuel [+ causatif] permet de distinguer, selon Baudet, Cordier et François (1997), une interprétation causative intentionnelle où l'agent accomplit une action par l'intermédiaire de laquelle il cause un changement, une interprétation causative non intentionnelle où l'agent est l'auteur d'une action qui cause un processus en dehors de son contrôle. En ce sens, cet Agent Causateur ressemble à une instance ou une force naturelle (le froid engourdit les doigts) qui ne peut avoir que le statut de Causateur non agentif. Une troisième interprétation possible est l'interprétation non causative et donc non intentionnelle que les auteurs illustrent avec l'exemple Marie s'est fait tuer dans un accident de voiture et où ils considèrent que Marie est un pur patient ${ }^{11}$. Ce point de vue n'est pas partagé par d'autres auteurs comme Tasmowski (1987), Gaatone (1976), etc. qui considèrent en général que le référent du sujet dans la forme factitive a toujours quelque chose d'actif. Nous y reviendrons (cf. 4.2).

D'un autre côté, nous pensons qu'il faudrait distinguer dans l'interprétation causative intentionnelle, celle où l'Agent Causateur accomplit une action ou un processus 
volontairement, qu'il contrôle parfaitement et par l'intermédiaire desquels il cause un changement, du cas où cet Agent Causateur n'est pas contrôleur du changement produit. C'est ce qui fait la différence entre la causation exprimée respectivement par les verbes détruire et causer déjà abordés. Si (27) ne porte aucune ambiguïté sur l'intentionnalité et la capacité de contrôle de l'Agent Causateur, (28) peut recevoir une interprétation intentionnelle ou non intentionnelle (Son fils lui a causé volontairement ou involontairement beaucoup de soucis) et dans les deux cas de figure, ce Causateur n'a aucun contrôle sur le changement produit.

En effet, le verbe détruire implique par son sens même qu'une action s'accomplit et que cette action est causatrice de changement ; donc le sujet humain est en même temps l'Agent d'une action contrôlée par lui et le Causateur d'un événement par cette action même. En revanche, pour causer, l'agentivité, et donc l'intentionnalité et la capacité de contrôle, n'est pas inscrite dans son sens même ; elle est prise en charge par un faire sousjacent, puisque le sujet humain de ce verbe ne se présente pas "en personne" comme porteur de la prédication. (28) correspond en réalité à la paraphrase : son fils a fait quelque chose (volontairement ou involontairement), ce qui lui a causé beaucoup de soucis. Avec ce verbe, on représente en réalité le rapport entre une action causatrice et l'effet de cette action.

51 Ce qui distingue donc à notre avis causer de détruire, et plus largement la causativité de l'agentivité, c'est la capacité de contrôle, c'est-à-dire, comme la définit Desclès (1990), la capacité de déclencher une action et de l'interrompre éventuellement avant son terme. Cette capacité est en effet constitutive de l'agentivité, mais non de la causativité ; alors que l'intentionnalité peut s'apparenter aux deux quand le sujet est bien entendu un humain. Un Causateur peut être caractérisé en effet comme une entité qui a la capacité de produire un changement, mais sans pouvoir le contrôler jusqu'à son terme. Il peut bien entendu le déclencher, le planifier, avoir une claire représentation du but visé et des actions qui permettent de l'atteindre, sans pour autant garantir un contrôle continu sur ce programme d'actions qui permettra de réaliser le changement tel qu'il est visé. Nous avons d'ailleurs remarqué que c'est cette caractéristique qui regroupe les différents types de Causateurs que nous avons examinés pour le verbe causer (cf. Kaheraoui, 2002), qu'ils soient des humains, des objets, des états, des événements ou des forces naturelles.

Mais on peut aussi concevoir les notions d'agentivité et de causativité comme des notions scalaires qui se manifestent par des degrés et non par des traits. En ce sens, comme le Causateur est selon nous par définition non contrôleur, il serait une sorte d'Agent "abâtardi". Cette conception scalaire a été soutenue par Desclès (1990) qui, tout en maintenant la distinction entre l'agentivité et la causativité, les repère sur des continuums distincts. Le continuum d'agentivité, qui implique une certaine capacité de contrôle et d'effectuation d'un processus, va de l'agent seulement contrôleur (Jean court dans le parc), à l'agent contrôleur et effectueur (Jean déplace les meubles), à l'agent contrôleur, effectueur et anticipant une action planifiée (Jean lance une pierre contre la voiture qui arrive). Le continuum de causativité met en évidence des zones catégorielles où la causativité peut s'exercer directement par un Agent Causateur sur un patient (Jean déplace la pierre), sur le processus qui fait entrer le patient dans un état (Julien endort Marie), sur l'action impliquant un agent secondaire (Julien fait marcher Marie) qui exerce lui-même une action sur un autre patient (Julien fait déplacer la pierre par le jardinier).

Desclès (1990) va cependant introduire la notion de "transitivité sémantique" qui joue le rôle d'un point tournant entre les continuums d'agentivité et de causativité. L'agentivité 
est constitutive de la transitivité sémantique (Hopper\& Thompson, 1980) et implique une certaine capacité de contrôle et d'effectuation d'un processus, de façon directe ou médiate. La causativité tient entre deux événements ou états dont l'un doit nécessairement être réalisé pour que l'autre puisse avoir lieu, alors que la transitivité sémantique est liée à un seul événement qui conduit à un état résultatif, avec deux participants, un agent qui a la capacité de contrôle et un patient affecté par cet état (Luc a cassé la tasse). Autrement dit, des verbes analysés comme étant causatifs (détruire, briser, casser, tuer, etc.) sont considérés comme des marqueurs de transitivisation, et donc d'expression d'une certaine agentivité ou encore d'un certain contrôle d'un actant sur un changement affectant un patient.

Dans ce modèle, la factitivité ne relève ni de la causativité, ni de la transitivité sémantique. Desclès la représente par un schème spécifique. Comme la transitivité, elle est relative à un seul événement, mais avec deux agents dont l'un est sous la dépendance de l'autre (Marie fait déplacer la pierre par le jardinier). Autrement dit, le verbe faire n'est pas un verbe causatif selon cet auteur, puisqu'il considère que dans la construction factitive, il y aurait un seul processus dans lequel sont impliqués trois participants : un agent principal qui a le contrôle sur le changement d'état produit et qui exerce un rôle d'instigateur ; un agent secondaire doté d'un certain pouvoir de contrôle qui lui permet d'exécuter ou non le changement voulu par l'agent principal et un patient affecté par ce changement. Selon Desclès (1998), il ne s'agit donc pas dans cette construction d'une relation causative entre deux événements, puisque l'agent principal n'est pas impliqué dans un événement qui serait la cause de l'événement verbalisé.

L'idée que l'argument-sujet de faire soit décrit comme un agent qui contrôle le changement produit ne peut pas être totalement défendue ; elle s'oppose d'emblée aux cas où le sujet est non humain comme dans l'exemple La fumée fait tousser. Mais ce cas, explique Desclès, n'exprime plus la factitivité, mais la causativité, puisque le lien dans cet exemple peut être exprimé entre deux situations où l'une est la cause de l'autre (Lorsqu'il y a de la fumée, on tousse). De même, la structure factitive peut encoder le schème de transitivité sémantique, comme dans Pierre fait craquer une allumette, puisqu'un seul agent est impliqué et que cet agent contrôle et agit sur un patient qui, lui, n'a aucune activité agentive.

Autrement dit, on n'a pas ici une analyse unifiée de la forme factitive ; selon les cas, elle peut s'apparenter à de la causativité, donc avec un sujet Causateur, ou à de la transitivité, avec un sujet Agent contrôleur. De même, il serait difficile d'admettre, quand faire +infinitif encode le schème de factitivité avec trois participants, que le sujet de faire n'est pas impliqué dans l'événement causateur de l'événement causé dans lequel est également impliqué le sujet de l'infinitif. C'est là toute la particularité de la forme factitive où il s'agit de définir le rôle respectif de ces deux sujets dans des rapports où se confronte la causativité du premier face à l'agentivité du second.

\subsection{Les rapports entre le sujet humain de faire (NP1) et le sujet de l'infinitif (NP2) dans la construction faire + infinitif}

\subsubsection{L'agentivité de NP2}

57 Nous avons proposé, en analysant les restrictions de sélection sur l'argment-sujet de faire, que la restitution d'une prédication elliptique est possible quand le sujet réfère à un 
humain, à condition que le verbe infinitif soit intransitif (voir les exemples 12-12a que nous reprenons ci-dessous). Mais quand le verbe est transitif et que le sujet de l'infinitif est introduit par les prépositions à ou par, cette restitution ne peut pas s'appliquer, comme le suggèrent (20) et (20a). On peut supposer alors que la présence d'un complément en par ou en à bloque ou rend superflue la présence d'un FAIRE sous-jacent. Pour en rendre compte, nous allons examiner l'agentivité du sujet de l'infinitif enchâssé, en rapport avec les constructions faire à et faire par.

(12) Le colonel Lejeune déboula sur la place et fit cabrer son cheval devant Masséna.

(12a) Le colonel Lejeune a fait quelque chose/a quelque chose en lui qui a la vertu de faire cabrer son cheval.

(20) Marie a fait balayer la cour par Pierre / à Pierre

(20a) *Marie a fait quelque chose/a quelque chose en elle qui a la vertu de faire balayer la cour par/à Pierre

Nous pouvons remarquer qu'en général, quand NP1 est un humain, on a tendance à considérer qu'il est un Agent, comme l'est le sujet enchâssé NP2. Langacker (1991 : 257) les désigne respectivement par "premier agent" et "second agent". Tasmowski-De Ryck \& Van Oevelen (1987 : 53) considèrent eux aussi que NP1 ne désigne une cause que quand il dénote une entité non animée. Pourtant, vu les types de sujets de faire qui jouent le rôle de Causateur, on aurait tendance plutôt à soutenir que le NP1 humain reçoit également ce rôle et que c'est sur son agentivité que planeraient des doutes.

L'agentivité de NP2 est en revanche plus convaincante. Nous avons vu que la représentation sémantique que font Zaenen \& Dalrymple (1996) de faire + infinitif, dans le cadre de la Grammaire Fonctionnelle, est indifférente au nombre d'arguments que peut prendre le verbe infinitif. Cette représentation requiert par contre que ce verbe ait un Agent. On peut arriver, peu ou prou, à la même constatation si on considère le cas des verbes transitifs dont les NP2 sujets se trouvent introduits par par ou par à dans la structure factitive. Le NP2 introduit par à est considéré comme un complément datif qui dénote le destinataire du procès ; il est alors préférentiellement un humain, comme l'illustrent (31) par rapport à (34a). Quant au NP2 introduit par par en (32), il est souvent rapproché des compléments d'agent qu'on retrouve dans la construction passive (33). En tant que tel, il ne dénote pas forcément des humains, comme le suggèrent (34/35), (36/37) :

(31) J'ai fait nettoyer la chambre à Pierre

(32) J'ai fait nettoyer la chambre par Pierre

(33) La chambre a été nettoyée par Pierre

(34) J'ai fait inonder les près par l'eau de la rivière

(34a)*J'ai fait inonder les près à l'eau de la rivière

(35) Les près ont été inondés par l'eau de la rivière

(36) On a fait enlever des échantillons par cette machine

(37) Des échantillons ont été enlevés par cette machine

(38)*Marie a fait nettoyer la chambre par un balai

On peut remarquer que le NP2 introduit par par peut être l'agent de l'action exprimée par le verbe infinitif (32), la cause (35) ou l'instrument utilisé pour l'accomplir (36). Mais cet instrument doit être doué d'une certaine motricité propre, comme le suggère l'agrammaticalité de (38). Plusieurs auteurs (Damourette \& Pichon, 1911-1936 ; Gaatone, 1976 ; Danell, 1979) se rejoignent en effet, en attribuant à par NP2 l'idée d'une intervention active. D\&P (Tome 3,530) considèrent notamment qu'avec par, le locuteur envisage NP2, " comme la cause efficiente, l'agent, l'outil » de la relation factitive à exprimer. Zubizarreta (1986 : 287), en se basant sur le rapprochement avec le passif, fait 
remarquer que le passif et la construction faire par peuvent tous les deux bloquer la réalisation syntaxique du rôle thématique externe du verbe qui les régit :

(39) L'architecte a fait tracer le plan méticuleusement

(40) Le plan a été tracé méticuleusement

61 Dans (39), le sujet sous-entendu enchâssé ne peut pas être interprété comme coréférentiel avec le sujet de faire. Il est interprété comme ayant une référence arbitraire, comme dans le cas des phrases passives sans syntagmes en par (40). Selon Zubizarreta, le rôle thématique d'Agent est présent lexicalement dans les passifs. C'est pour cela que ceux-ci peuvent apparaître avec des adverbes (méticuleusement) qui modifient des verbes qui contiennent un argument susceptible d'être interprété comme agentif et c'est le même cas avec la construction faire par en (39).

Par rapport à par NP2, on peut noter que le complément à NP2 réfère exclusivement à des humains. Ainsi, l'ordre d'apparition de ces deux prépositions est parfois contraint ; il dépend aussi bien de la nature de NP2 que de celle de NP1. Quand NP2 ne dénote pas un individu humain, mais une entité douée d'une certaine motricité, et que NP1 désigne un humain, le choix porte sur par (34), plutôt que sur à (34a). A l'inverse, quand NP2 est un humain et NP1 non-humain, le choix portera sur à plutôt que sur par. C'est ce qu'illustrent $(41,42)$ par rapport à $(41 \mathrm{a}, 42 \mathrm{a})$ :

(41) La famine a fait manger des rats aux habitants de la ville

$(41 \mathrm{a}){ }^{*}$ La famine a fait manger des rats par les habitants de la ville

(42) Il s'en souvenait quand cette vieille douleur lui faisait porter une main à la nuque

(42a)*Cette vieille douleur faisait porter une main à la nuque par Paul ${ }^{12}$.

Nous pensons que le choix de à ou de par correspond à une interprétation sémantique de la relation causative dans laquelle l'emploi de ces deux prépositions permet d'expliciter la relation sémantique assumée par NP2, le sujet de l'infinitif, vis-à-vis du Causateur NP1 dénotant un humain.

\subsubsection{Faire par et faire à : rapport entre NP1 et NP2}

Nous avons vu que Kayne (1977) résout le problème de l'interprétation appropriée des NP2 postverbaux, dans la construction faire + infinitive, par l'application des transformations FI/INS-A pour les constructions en faire à et FP pour les constructions en faire par. Morin (1978) réfute les arguments invoqués par Kayne dans la mesure où, selon lui, on n'utilise les transformations que pour capturer des généralisations de type syntaxique et non sémantique. Pour cet auteur, le problème de l'interprétation de la position postverbale des NP2 sujets dans la forme factitive est en effet essentiellement un problème sémantique. Ainsi, si comme le propose Kayne, les constructions factitives étaient engendrées par des variantes de la même règle, on s'attendrait à ce qu'elles aient toutes le même sens. Or, les constructions en faire à et celles en faire par ne sont pas synonymes. Dans les exemples (43) et (44), les contextes entre parenthèses font ressortir, selon Morin (1978: 395), l'interprétation différente des deux constructions :

(43) L'entraîneur fait courir le 100 mètres à son fils (pour lui fortifier les jambes)

(44) L'entraîneur fait courir le 100 mètres par son fils (parce que son meilleur coureur est blessé)

Dans la construction faire à, le NP2 est interprété non seulement comme agent du verbe courir mais surtout comme bénéficiaire de faire, alors que dans la construction faire par, le NP2 est interprété comme agent du groupe V-NP (courir le 100 mètres), mais n'a pas de 
fonction par rapport à faire. Rappelons que Morin considère que faire forme avec l'infinitif un verbe complexe. Par conséquent, son analyse soutient que par NP2 et à NP2 n'occupent pas la même position structurale : le groupe en par appartient à la proposition enchâssée, alors que à NP2 est sous-catégorisé par le verbe faire. Le complément en à fonctionnerait alors comme un vrai datif, le troisième actant d'un verbe (faire + infinitif) trivalent.

On peut remarquer que même si Morin affirme que le problème de ces NP2 est d'abord sémantique, il ne donne ici qu'une solution apparentée à leur position structurale. Damourette et Pichon proposent en revanche de faire correspondre le choix de à ou par à deux interprétations sémantiques qui mettent en rapport le Causateur NP1 et le sujet enchâssé NP2 : «Si le sentiment linguistique conçoit l'entrejet surtout comme intéressé dans le phénomène global, l'entrejet-agent apparaît sous le rayon à » (Damourette et Pichon, § 1071, Tome 3, p. 522). Mais « si le sentiment linguistique conçoit l'entrejet surtout comme ayant un rôle actif en tant qu'il est l'agent de l'infinitif conspicience, l'entrejet apparaît sous le rayon par »(§ 1078, p. 529). Les auteurs présentent donc l'alternative entre les deux prépositions comme une opposition sémantique : avec à, l'entrejet, c'est-à-dire NP2, est envisagé " comme intéressé dans le phénomène », alors qu'avec par, il est envisagé « comme la cause efficiente, l'agent, l'outil de ce phénomène » (§ 1080, p. 530).

67 Cela veut dire, en illustrant par les exemples (45) et (45a), que la fin principale que l'on envisage en (45), au moins au moment où la phrase est énoncée, c'est de faire que l'interne opère, de lui donner l'occasion de perfectionner sa virtuosité chirurgicale. En revanche, dans (45a) avec par, la fin principale qui est présentement pensée, c'est que le malade soit opéré et par là débarrassé de son mal ; savoir qui sera l'agent de l'opération est un point accessoire, l'interne apparaît en quelque sorte comme l'outil qu'emploie le chirurgien pour que l'opération soit faite :

(45) Le chirurgien fit opérer ce malade à son interne

(45a) Le chirurgien fit opérer ce malade par son interne

68 Autrement dit, dans faire à, le Causateur NP1 ne tend pas seulement à ce qu'un événement ait lieu, à ce qu'une action s'accomplisse, il tend vers l'obtention d'une situation pour autant qu'elle implique NP2 et le concerne. En d'autres termes, l'événement déclenché par ce NP1 dans le chef de NP2 a comme effet que NP2 en souffre une certaine conséquence ; il est amené à une forme d'action qui peut être aussi bien à son avantage qu'à son détriment. L'argument marqué par à est alors défini d'abord comme affecté par la causation ${ }^{13}$. Cela expliquerait alors pourquoi, quand NP1 est un événement ou une entité non animée, le complément en à est préféré à celui en par, comme l'illustrent les exemples (41) et (42).

En revanche, avec par, l'attention de NP1 est portée sur l'action dénotée par l'infinitif, peu importe qui en est l'agent. Ce qui expliquerait pourquoi le complément en par dans faire + infinitif peut ne pas être réalisé syntaxiquement. Tasmowski-De Ryck \& Van Oevelen (1987 : 52) arrivent à expliquer par le même type d'analyse, les jugements de grammaticalité dont la construction factitive fait l'objet, selon que NP2 est introduit par à ou par par. A la différence de par NP2 considéré comme un "point accessoire", le complément à NP2 ne saurait être facultatif. C'est pourquoi, toute phrase factitive, si elle était "complète", serait de la forme faire par. Et toute phrase factitive grammaticale sans la réalisation d'un complément en par ou en à, doit rester grammaticale sous la forme faire par (46-46a), mais peut devenir agrammaticale sous la forme faire à (46b) : 
(46) On vous fera téléphoner le résultat

(46a) On vous fera téléphoner le résultat par le secrétaire

(46b)* On vous fera téléphoner le résultat au secrétaire

l'action dénotée par l'infinitif, avec faire à, NP2 doit être atteint par NP1 et ne doit donc bénéficier à son égard que d'une relative autonomie. Par conséquent, plus l'atteinte de NP2 par NP1 est garantie, plus à NP2 sera naturel. Parallèlement, plus l'intérêt va à l'accomplissement de l'action dénotée par l'infinitif, c'est-à-dire le phénomène causé, plus par NP2 sera adéquat. C'est ainsi que Tasmowski-De Ryck \& Van Oevelen (1987 : 52) interprètent l'absence de à NP2 dans la forme factitive pronominale. En effet, dans cette forme, par NP2 est presque toujours obligatoire :

(47) Marie se fait conduire par Paul

(47a)*Marie se fait conduire à Paul

Dans ce tour causatif pronominal, se renvoie au sujet global du factitif (Marie). C'est ce sujet qui met en branle un processus qui l'affecte ; rien ne saurait donc être en principe plus digne d'intérêt que ce qui lui arrive. C'est pour cela que l'attention est dirigée d'une manière inhérente vers l'accomplissement de l'action, vers l'obtention d'une situation et NP2, l'instrument de cette obtention, ne saurait s'exprimer qu'à l'aide de par NP2.

\subsubsection{La causativité de NP1 face à l'agentivité de NP2}

Comme les rapports entre NP1 et NP2 changent selon que NP2 est introduit par à ou par par, nous proposons d'examiner comment ces rapports s'interprètent en terme de causation ; autrement dit, comment NP1 « exerce-t-il » sa causativité.

Nous pensons que l'explication passe par la notion de contrôle que nous considérons, avec une valeur négative, comme un trait définitoire de la causation. C'est cette notion d'ailleurs qui permet d'expliquer le recours à la construction factitive, considérée comme indirecte, par rapport à la construction causative directe correspondante. Dans la mesure où la causation représentée par la forme factitive s'exerce sur des actions qui impliquent des Agents, on peut comprendre qu'elle soit décrite comme une causation indirecte par rapport à la causation exprimée par les verbes causatifs simples correspondants. Ainsi, on considère que la causation s'exerce directement sur un patient en (48a-49a), alors qu'elle s'exerce sur l'action impliquant un agent qui exerce lui-même cette action sur un patient en (48-49) :

(48) Jean a fait remonter Marie sur un mur

(48a) Marie a remonté sur un mur

(49) Jean a fait déplacer la pierre par Marie

(49a) Marie a déplacé la pierre

C'est ainsi que Ruwet (1972:159) explique le caractère anormal de (50) par rapport à (50a), parce qu'il est impossible de considérer la fusion du sucre dans le café comme le résultat d'une action directe du colonel sur le sucre :

(50) *Le colonel a fondu trois sucres dans son café

(50a) Le colonel a fait fondre trois sucres dans son café

(51) Le métallurgiste a fait fondre le métal

(51a) Le métallurgiste a fondu le métal

Cette distinction est mise en relation avec la présence ou l'absence d'un contrôle continu $\mathrm{du}$ Causateur NP1 sur le processus auquel est soumis NP2, c'est-à-dire trois sucres, et qui correspond à l'objet du verbe causatif simple en (50). Dans la forme factitive, la part du 
colonel dans le processus de fusion tient seulement au fait qu'il ait mis le sucre dans son café ; la fusion du sucre tient à des causes qui échappent à son contrôle. Bien entendu, le NP2 trois sucres ne peut être tenu pour un agent, mais il participe au processus de fusion de manière autonome qui échappe au contrôle de NP1. Pour Ruwet, il en va autrement pour (51) où dans les deux cas, le sujet Causateur garde un contrôle continu et direct sur l'opération de fusion du métal. La question qui se pose alors est pourquoi en (51a) le métallurgiste peut agir directement sur le métal et contrôler son processus de fusion, alors qu'en (50), le colonel n'a pas ces capacités ?

76 Wierzbicka (1988 : 244) se pose la même question, puisqu'en traduisant ces exemples en anglais, elle constate que seul le causatif lexical simple est utilisé, que ce soit pour (50)/ (52) ou pour (51)/(53) :

(52) The colonel dissolved three lumps of sugar in his coffee

(52a)*The colonel made three lumps of sugar dissolve in his coffee

(53) The metallurgist melted the metal

Selon cet auteur, la raison pour laquelle la forme factitive avec make est incorrecte en (52a) est que le Causateur peut agir directement sur un causataire (causee) inanimé (i.e. le sucre). Sans vouloir donner des pouvoirs "supérieurs" au colonel "anglais", Wierzbicka pense qu'en anglais, la causation indirecte des factitives suppose que lorsque le Causateur fait quelque chose, il ne fait rien à NP2 avant que le changement escompté se produise, alors que les factitives en français supposent qu'avant que le changement escompté se produise, il y a un processus autonome qui intervient au niveau de NP2, distinct à la fois de l'action du Causateur sur NP2 et du changement final produit qui affecte ce dernier. Cela veut dire donc que le sucre, quand il est placé dans un café chaud, subit un processus interne semi-autonome de fusion. C'est justement ce processus qui échappe au contrôle du Causateur. Quant au métallurgiste, on peut imaginer qu'il puisse agir directement sur le métal et contrôler son processus de fusion, parce qu'il peut le mettre sous des conditions expérimentales particulières, par exemple le maintenir sous une certaine température ou dans un produit spécial pour le fondre. En même temps, il peut arrêter ce processus de fusion en suspendant ces conditions. C'est en ce sens qu'il faudrait interpréter (51a). L'interprétation change pour la forme faire + infinitif de (51). Elle suppose en effet que le métallurgiste n'a pas agi directement sur le processus de fusion du métal ; il l'a fait fondre soit par quelqu'un, soit par une entité qui a la vertu de faire fondre des métaux. En tout cas, le Causateur agit en tant que déclencheur d'un processus dont il perd le contrôle par la suite. Il intervient en effet un élément de durée dans la réalisation du changement qui fait que la réalisation de l'effet désiré, c'est-à-dire la fusion du métal, ne dépend plus du Causateur, mais du métal lui-même dont l'action du Causateur a modifié l'état.

Autrement dit, si la causative lexicale simple attribue la causation uniquement au Causateur, puisqu'il est à la fois Agent et Causateur, la structure avec faire + infinitif attribue la causation en partie au Causateur NP1, en partie à NP2. La construction factitive implique que le Causateur active un processus, notamment ici dans un NP2 inanimé (sucre, métal) qui déclenche alors le résultat final. Nous avons déjà vu que NP2 est présenté comme étant plus ou moins agentif, qu'il soit marqué par à ou par par, dans la mesure où il a un rôle actif. Dans ces exemples, le sujet enchâssé NP2 est inanimé, mais il est pourvu d'une activité propre, ce qui lui permet de participer à la causation. C'est cette caractéristique qui permet à NP2 de remplir son rôle de " pivot causatif ", selon les 
termes de Langacker (1991), c'est-à-dire qu'il fonctionne comme la "trajectoire" de la prédication représentant l'activité causée.

Ce pivot est donc à un certain degré responsable de la production du changement escompté. En même temps, ses efforts sont eux-mêmes induits par le Causateur NP1 situé en haut de la chaîne d'actions. NP2 se présente donc à la fois comme participant à la causation et comme subissant le processus dirigé par une énergie externe. On peut alors se demander si l'introduction des prépositions à ou par influence le degré de participation de ce NP2 à la causation du changement.

Langacker note que, dans certaines langues comme le japonais ${ }^{14}$, le choix entre l'accusatif et le datif pour marquer le sujet enchâssé NP2 n'est pas neutre d'un point de vue sémantique. L'accusatif est utilisé quand le Causateur est indifférent à ce que NP2 soit ou non consentant, alors que le datif implique que NP2 agit volontairement. Rappelons que l'intentionnalité d'un sujet n'implique pas son agentivité ; elle implique seulement un certain degré d'agentivité. Quant au contrôle, qui est un trait constitutif de l'agentivité, nous pensons que rien ne spécifie en français que le NP2 datif a ce pouvoir sur l'événement dénoté par l'infinitif.

81 A propos des constructions causatives en faire à, qui peuvent prendre aussi bien le clitique datif (54a) que le clitique accusatif (54b), Authier \& Reed (1991) considèrent également que l'individu dénoté par le clitique accusatif n'a pas eu d'autre choix que de manger des épinards, alors qu'en (54a), ce sujet marqué par le datif peut aussi bien avoir eu le choix que non :

(54) J'ai fait manger des épinards à Maurice

(54a) Je lui ai fait manger des épinards

(54b) Je l'ai fait manger des épinards

Selon ces auteurs, si (54b) exprime une « causation directe », (54a) peut exprimer à la fois une causation directe ou indirecte. Pour expliquer ces inférences, les auteurs font appel à la notion de contrôle sémantique du NP2 sur l'événement dénoté par le prédicat enchâssé. Pour eux, l'individu dénoté par le clitique accusatif n'a aucun contrôle sur l'événement dénoté par le verbe infinitif, alors qu'avec le datif on n'en dit rien. Le fait qu'une phrase avec un NP2 marqué au datif (54) puisse être interprété doublement selon le type de clitique suggère à notre avis l'incertitude qui plane sur le degré de contrôle qu'exerce NP2 sur l'événement dénoté par l'infinitif. En fait, ce NP2 est présenté essentiellement comme l'argument affecté par le processus.

Dans la liste des rôles thématiques, Langacker (1991) présente l'Experiencer comme l'archétype qui fournit la valeur protoypique du datif. Rappelons également, que dans cette liste, le rôle thématique d'Experiencer se positionne juste derrière l'Agent, ce qui reflète la capacité de ce participant à fournir une source d'énergie. En effet, dans la catégorie des récepteurs, l'Experiencer est un participant actif. Donc, en marquant le NP2 au datif dans la structure factitive, ce NP2 fonctionne à la fois comme affecté par le processus causatif et comme un intermédiaire actif qui participe à ce processus. En ce sens, il n'est pas Agent, au sens de déclencheur d'une action qu'il contrôle de manière continue, mais seulement doué d'un certain degré d'agentivité. En tant que destinataire du changement produit, le NP2 introduit par à doit être atteint par le Causateur et en ce sens il ne peut jouir de sa part que d'une relative autonomie.

Quant au NP2 marqué par par, en le caractérisant comme un instrument, l'outil utilisé par le Causateur NP1 pour produire un changement, il est considéré aussi comme ayant un degré d'agentivité. A-t-il un plus grand degré d'agentivité que à NP2 ? Selon la hiérarchie 
des cas de Fillmore (1975), l'Instrument vient après l'Experiencer. Mais en considérant la relation entre les rôles thématiques, Langacker (1991 : 260) fait remarquer que l'instrument est considéré comme une extension de l'Agent et appartient donc au domaine des rôles thématiques-source, alors que l'Experiencer fait partie des rôles thématiques récepteurs. Nous avons vu qu'il est rare de trouver un Causateur non humain dans les structures faire à et faire par. Quand c'est le cas comme dans l'exemple (55) que nous reprenons ci-dessous, NP2 est marqué au datif. En revanche, le complément en par est exclu (55a) :

(55) La famine a fait manger des rats aux habitants de la ville

(55a)*La famine a fait manger des rats par les habitants de la ville

Cela peut s'expliquer par le fait que ce NP2 introduit par par a un degré d'agentivité plus important que le complément en à. Comme on l'a déjà démontré, ce complément est présenté essentiellement comme affecté par le processus de causation. Ce qui confirmerait donc la primauté de la catégorie des rôles-sources, à laquelle appartient par $\mathrm{N} 2 \mathrm{P}$, sur celle des rôles-récepteurs à laquelle appartient à NP2.

Nous proposons donc de caractériser le Causateur humain argument-sujet de faire comme une instance causatrice ou une force extérieure qui déclenche un processus causateur, mais qui met en jeu nécessairement des entités douées d'un degré plus ou moins grand d'agentivité. En effet, ces entités participent d'un côté à la production du changement escompté en tant qu'entités autonomes, d'un autre côté, elles subissent le processus de causation dirigé par le Causateur dans la mesure où celui-ci exerce une sorte de coercition sur elles. C'est cette coercition qui déclenche d'ailleurs leur propre action. Nous pouvons alors imaginer que plus la coercition exercée par le Causateur sur NP2 est grande, moins ce NP2 participe à la causation, c'est-à-dire qu'il serait moins agentif. Ainsi, les NP2 marqués à l'accusatif seront considérés comme moins agentifs que les NP2 marqués au datif, lesquels sont eux-mêmes moins agentifs que les NP2 marqués par par.

Ce qui fait donc la particularité de la construction en faire dans l'expression de la causation, c'est le rôle du NP2 qui bénéficie d'une certaine autonomie, que le Causateur soit humain ou non d'ailleurs. En fait, dans cette construction, l'activité de la partie, qui engage NP2, constitue en même temps l'activité du tout, c'est-à-dire celle qui engage NP1 dont le but est de produire un changement. Ainsi, il ne peut pas y avoir simultanéité entre l'activité partielle et le but poursuivi. Force est alors de conclure à une distance indispensable entre l'action du Causateur NP1 et la production de changement. Cette distance se traduit à notre sens par la perte de contrôle du Causateur NP1 sur le changement produit. La présence de NP2 ainsi que le rapport établi entre NP2 et NP1 montrent que la causation de changement ne dépend pas uniquement de NP1, mais aussi de NP2. La construction factitive implique donc une phase où le Causateur devient passif, le relais étant pris par NP2, dont l'action est à la fois distincte de celle du Causateur et du changement final produit. Ce relais peut être pris par la situation provoquée elle-même si NP2 est une entité inanimée (sucre, métal, bois etc.), mais qui reste active dans la mesure où elle entre dans un processus semi-autonome avant le résultat final. Quand NP2 dénote un humain, c'est en fonction de son degré d'agentivité qu'est mesurée sa part de participation : plus cette part est grande, moins la contrainte que NP2 subit de la part du Causateur est importante.

Nous pouvons alors revenir sur les restrictions de sélection du sujet humain dans faire +infinitif et imputer l'agrammaticalité des paraphrases proposées de l'exemple (20) que 
nous reprenons ci-dessous en (56) et (56a) à l'agentivité des compléments introduits par les prépositions à et par :

(56) Marie a fait balayer la cour par Pierre / à Pierre

(56a) *Marie a fait quelque chose/a quelque chose en elle qui a la vertu de faire balayer la cour par/à Pierre

(57) Jean a fait pleurer Marie

(57a) Jean a fait quelque chose (volontairement ou involontairement), ce qui a fait pleurer Marie

(57b) La stupidité de Jean a fait pleurer Marie

(58) Jean a fait porter Pierre un rocher

(58a) Jean a fait quelque chose (volontairement ou involontairement), ce qui a fait porter Pierre un rocher

(58b) La colère de Jean a fait porter Pierre un rocher

Dans la structure factitive où NP2 est marqué à l'accusatif, que le verbe soit d'ailleurs intransitif (57) ou transitif (58), on peut stipuler l'existence d'un prédicat elliptique qui dénote une action effectuée par le Causateur (57a-58a) ou une propriété qu'il porte (57b-58b). NP2 agit comme un animé qui a un degré minimal d'agentivité : il est agent seulement dans la mesure où il exerce une action (pleurer, porter), mais il n'a pas de jugement indépendant sur cette action, ni de volonté propre. Si Jean fait pleurer Marie volontairement, le jugement d'intentionnalité ne se rapporte pas à Marie, mais à Jean ou plus précisément à l'action sous-jacente accomplie par lui. Ce que montre la paraphrase proposée. Dans ces tours, le Causateur est seul maître de la situation ; NP2 apparaît comme subissant complètement le processus déclenché par le Causateur NP1. En revanche, quand NP2 est introduit par à ou par par (56), ce qui bloque la reconstruction du prédicat elliptique, c'est la participation effective des compléments introduits par ces propositions à la causation. NP2 est présenté comme étant plus actif dans le processus de causation, avec les différences de degrés que nous avons expliquées quand la préposition est à ou par.

\section{Conclusion}

La causation exprimée par le lexique verbal est très riche. Les distinctions résident dans le mode d'expression de la causation au niveau de l'argument-sujet en relation avec l'agentivité et, dans le mode de production de changement saisi au niveau de l'argumentobjet. Nous avons abordé les exemples des verbes de type détruire et causer en comparaison avec faire. Alors que détruire demande un sujet Causateur qui est aussi un Agent contrôleur de l'action accomplie et un objet-patient affecté par le changement produit, causer fonctionne comme un relateur entre un événement causé par un sujet Causateur non contrôleur et l'expression du changement subi par un patient. Entre ces deux catégories de verbes, la particularité de faire+infinitif est que la causation exprimée passe par un pivot causatif intermédiaire, le sujet de l'infinitif, qui participe donc au processus de causation et à la production du changement dont il est lui-même affecté. Ceci dit, il conviendrait d'étendre l'analyse, pour mieux l'affiner, à d'autres verbes et structures causatives et pour le cas de faire+infinitif, c'est la comparaison avec laisser +infinitif et, éventuellement leur relation avec obliger, contraindre, permettre, empêcher, etc., qui serait encore plus instructive quant au fonctionnement de cette forme factitive dans l'expression de la causation. 


\section{BIBLIOGRAPHIE}

Authier, J.-M. \& Reed, L. (1991) : « Ergative predicates and dative cliticization in French causatives », Linguistic Inquiry, 23. 197-205.

Baudet, S., Cordier, F., François, J. (1997) : «L'identification des classes de procès en contexte II : étude expérimentale », J. François et G. Denhière éd., Sémantique linguistique et psychologie cognitive. Aspects théoriques et expérimentaux, Grenoble : Presses Universitaires de Grenoble. 249-282.

Damourette, J.\& Pichon, E. (1911-1940) : Des mots à la pensée : Essai de grammaire de la langue française, Paris : D'Artrey, 7 Tomes.

Desclés, J.-P. (1990) : Langages applicatifs, langues naturelles et cognition, Paris : Hermès.

Desclés J.-P, Guentchéva Z. (1998), "Causalité, Causativity, Transitivity", Typology of verbal Categories, ed. L. Kulikov, H. Vater.7-27.

Dowty, D. (1991) : « Thematic proto-rôles and argument selection », Language 67. 574-619.

François, J. (1989) : Changement, causation, action, Genève : Droz.

François, J. (1996) : « En deça, au terme et au delà de la table ronde : l'agentivité, la causativité, l'inadvertance et la diversité des langues ", Scolia, 7. 255-274.

Kaheraoui, M. (2002) : "La structure argumentale du verbe causer et caractérisation du concept grammatical de cause", Travaux linguistiques du CERLICO, n¹5. 191-209.

Kayne, R. (1977) : Syntaxe du français : le cycle transformationnel, Paris : Le Seuil.

Khalifa, J.-Ch. (2006) : "Pour une cartographie des causatifs en anglais contemporain". Corela, volume $4, n^{\circ} 1$.

Langacker, R. (1991) : Concept, image, and symbol. The cognitive basis of grammar, Berlin. New york : Mouton de Gruyter.

Lazard, G. (1994) : L'actance, Paris : Presses Universitaires de France.

Morin, J.-Y. (1978) : « Une théorie interprétative des causatives en français », Lingvisticæ Investigationes, II : 2. 363-417.

Reed, L. (1991) : « The thematic and syntactic structure of french causative constructions », Probus, 33. 317- 360 .

Rœgiest, E. (1982-83) : « “A” et “par” dans la construction factitive : L'EGLF dans la perspective de la linguistique contemporaine », Travaux de linguistique, 9-10. 127-143.

Ruwet, N. (1972) : Théorie syntaxique et syntaxe du français, Paris : Seuil.

Ruwet, N. (1990) : « Des expressions météorologiques », Le français moderne, 1 / 2. 43- 97.

Tasmowski, L. et Van Oevelen, H. (1987) : « Le causatif pronominal », Revue romane, 22 : 1. 40-58.

Vendler, Z. (1967) : Linguistics in philosophy, Ithaca, New York : Cornell University Press.

Wierzbicka, A. (1988) : The semantics of grammar, Amsterdam/Philadelphia : John Benjamins Publishing Company. 
Zaenen, A. \& Darlymple, M. (1996) : « Les verbes causatifs polymorphiques : les prédicats complexes en français » Langages, 122. 87-95.

Zubizarreta, M.-L., (1986) : « Le statut morpho-syntaxique des verbes causatifs dans les langues romanes » Couquaux, D., Ronat, M. éd., La grammaire modulaire, Paris : Minuit. 279-311.

\section{NOTES}

1. Le terme "factitif" est utilisé plutôt dans la linguistique française ; dans la linguistique anglosaxonne, on utilise le terme "causatif" même pour les verbes traduisant faire, essentiellement make (Gilquin, 2006). D'autres auteurs préfèrent garder la distinction en français entre ces deux termes. Lazard (1994: 164, note 1) appelle causatifs les tours dérivés de verbes intransitifs "faire tomber quelqu'un" et factitifs ceux qui sont dérivés de verbes transitifs "faire voir quelque chose à quelqu'un". Desclès (1990) distingue lui aussi la causativité de la factitivité en les représentant par des schèmes différents.

2. Ruwet $(1990: 58)$ note que la relation entre pleuvoir et pluie ressemble à celle qui existe généralement entre les verbes et les dérivés nominaux correspondants. Et en note de page $\left(\mathrm{n}^{\circ}\right.$ 25), il explique que la tendance est de dire que ce sont les verbes météorologiques qui sont dérivés des noms correspondants et non l'inverse. Mais il cite des auteurs comme Bloch et von Wartburg (1975) qui affirment que la plupart des noms météorologiques dans les langues romanes ne dérivent pas directement des noms latins correspondants, mais qu'ils ont été construits sur la base des verbes romans. Ils donnent l'exemple du verbe tonner qui est plus fréquent dans les dialectes romans que le nom tonnerre. Cependant, beaucoup de noms météorologiques ou temporels n'ont pas de correspondants verbaux impersonnels : aube, crépuscule, averse, tremblement de terre, tempête, orage, ouragan...

3. Martin (1996 : 43) désigne les noms comme mot, discours, texte, ouvrage, etc., par « objets symboliques » vu qu'ils sont concrets et abstraits à la fois : concrets par leur signifiants et abstraits par leur signifié ; concrets par leur substance et abstraits par leur forme. Avec ce type de noms, la langue utilise en fait le même mot pour désigner aussi bien le résultat d'une production intellectuelle, c'est-à-dire un objet " idéal ", que le support matériel qui le rend accessible.

4. L'un des problèmes posés par des phrases à structure factitive comme $(20,21,22)$ est la position postverbale du NP sujet de l'infinitif. Selon Morin (1978 : 363), ce problème à proprement parler consiste à dire que puisque le NP ne précède pas mais suit le verbe auquel il doit être rattaché sémantiquement, les règles qui interprètent un NP en position préverbale comme remplissant telle ou telle fonction sémantique par rapport au verbe ne peuvent pas s'appliquer.

5. Dans un deuxième temps, Kayne (1977) a postulé que la dérivation de faire par serait facultative, à condition que la passif soit décomposé en deux règles moins complexes. L'une dite d'Agent postposing (postposition d'Agent) déplace le sujet profond en position de complément d'agent ; l'autre dite de NP-preposing (antéposition de NP) déplace l'objet profond en position de sujet en même temps que l'auxiliaire du passif est introduit. Seule la première de ces deux règles intervient dans la dérivation de faire par étant donné que le NP objet profond reste à droite du verbe et que l'auxiliaire ne s'introduit pas.

6. Cf. Chomsky $(1981,1982)$.

7. Rappelons que le $\theta$-rôle externe est assigné à un argument dans la position [NP, S] d'une phrase. Le $\theta$-rôle interne est assigné à un argument à l'intérieur de VP (cf. Zubizarreta, 1986 : 280). 
8. L'auteur a fourni d'autres arguments à l'encontre de l'analyse de faire + infinitif comme un verbe complexe du point de vue thématique (voir Reed, 1991).

9. Cf. Abeillé, 1993, Bresnan, 2001 pour une version récente.

10. L'Agent prototypique de Dowty présente un ensemble de cinq propriétés : a) engagement volontaire dans l'événement ou l'état, activité sensorielle et / ou perceptive, causation d'un événement ou d'un changement d'état sur un autre participant, mouvement (relatif à la position d'un autre participant), existence de l'entité désignée par l'argument indépendamment de l'événement désigné par le verbe.

11. Selon François et al. (1997), si Marie peut être analysée comme un pur patient, c'est parce que le français dispose d'une construction au passif (Marie a été tuée dans un accident de voiture) qui est sémantiquement équivalente à cette construction factitive. Ainsi, quand la forme passive n'est pas possible, comme dans *Marie a été arraché(e) son sac par un voyou, c'est la construction factitive correspondante (Marie s'est fait arracher son sac par un voyou) qui lui est substituée. Donc, selon ces auteurs, la construction factitive, qui présuppose à l'origine une participation causative, perd cette présupposition quand elle fonctionne comme un substitut de la construction passive.

12. Notons que (42a) illustre le cas où l'objet du verbe infinitif renvoie à une partie du corps (une main) interprétée comme une possession inaliénable du sujet. C'est en effet un contexte qui est défavorable aussi bien à la mise au passif qu'à la construction en faire par. On énumère d'autres contextes où la construction faire par comme le passif et à la différence de faire à, ne sont pas possibles comme les expressions idiomatiques $\left({ }^{*} \mathrm{il}\right.$ a fait casser sa croûte par sa famille/à sa famille) ; les verbes avec un objet locatif (*Je ferai quitter ma maison par Jean/à Jean) (cf. Kayne, 1977).

13. C'est l'interprétation proposée par Roegiest (1982-83).

14. C'est également le point de vue de Shibatani (1982), rapporté par Lazard (1994: 188-189), à propos de l'exemple : watasi-wa kodomo-o/kodomi-ni dan kara traduit par je fis descendre l'enfant du quai. L'emploi du datif (ni) suggère que l'enfant a une certaine part dans la décision, alors que l'accusatif (o) implique que seule la volonté du causateur est en cause.

\section{RÉSUMÉS}

Nous proposons dans cet article d'analyser la structure argumentale de la forme "faire+infinitif" en mettant en relief son comportement "bivalent" aux niveaux syntaxique et sémantique. Cette forme suggère en effet deux lectures syntaxiques différentes : l'une considère que faire est un verbe autonome qui prend pour argument-objet une proposition enchâssée infinitive ; l'autre soutient au contraire que faire est un auxiliaire d'un type spécial qui se combine avec le verbe infinitif afin de former un verbe complexe. En nous basant sur l'analyse de la grammaticalisation de la causation exprimée par les rôles thématiques, nous tenterons de rendre compte de la spécificité de cette forme, en comparaison avec d'autres verbes causatifs, dans l'expression de la causation et de la production du changement affectant un patient.

In this article, I propose to analyse the argument structure of the form "faire + infinitive" by highlighting on its "bivalent" conduct on a syntactic and semantic levels. In fact, this form offers two possible readings on a syntactic point of view. One is to consider that "faire" is an autonomous verb, which includes an infinitive clause as an object ; the other is to say that "faire" 
is a particular auxiliary which combining itself with the infinitive of the verb turns it into a more complex one. By taking support on the analysis of the grammaticalisation of the causation which is expressed by the sets of themes roles, I will try to debrief on the specificity of this form as compared to other causative verbs, in the expression of the causation and the production of the change assigning a patient.

INDEX

Mots-clés : causatif, factitif, contrôle, structure argumentale, changement d'état Keywords : causative, factitive, control, argumental structure, change of state

\section{AUTEUR}

\section{MALIKA KAHERAOUI}

IUFM de Poitiers, Laboratoire FORELL 